\title{
The Relationship of Clinical Biobank and Clinical Disciplines: A Survey Among Medical and Scientific Professionals in Hospital of China
}

\author{
Yang Wang ${ }^{1}$, Zheng Xiang ${ }^{1}$, Yue $\mathrm{Lu}^{1}, \mathrm{Min}_{\mathrm{Cao}}{ }^{1}$, and Yao Zhao ${ }^{1}$ \\ ${ }^{1}$ Chongqing Medical University Affiliated Children's Hospital
}

May 5, 2020

\begin{abstract}
Objectives: Clinical biobank is an important way to collect biological samples of various diseases, which is of great significance for clinical research. However, the relationship of clinical biobank and clinical disciplines still need to be further explored, thus we conducted a survey among medical and scientific professionals in a hospital of China. Method: The survey was conducted online, and electronic questionnaires were used to investigate knowledge, utilization and demand of biobank of medical and scientific professionals in different clinical disciplines. In terms of data analysis, chi-square test and rank sum test were used to analyze the collected data. Results: A total of 182 valid questionnaires were collected. The overall survey participation rate of key disciplines is higher than that of non-key disciplines. Key disciplines have higher score on the knowledge of biobank than non-key disciplines. The proportion of sample storage and sample delivery of key disciplines are far higher than non-key disciplines, and key disciplines have higher sample delivery rate than non-key disciplines. The proportion of people who are willing to pay to store samples and people who are willing to pay more than 5000 yuan per year for sample storage in key disciplines are higher than that in non-key disciplines. The per capita national grant application, the approval rate of national grant and per capita published English papers of key disciplines are higher than those of non-key disciplines. Conclusion: Key disciplines are more enthusiastic about the construction of biobank. On the whole, the knowledge, utilization and demand on biobank of key disciplines are better than those of non-key disciplines. The development of key disciplines is better than that of non-key disciplines. The development of clinical biobank is closely related to the development of clinical disciplines, and clinical biobank is an important booster for the development of clinical disciplines.
\end{abstract}

\section{Introduction}

According to the definition of the Organization for Economic Cooperation and Development (OECD) in 2001, the biobank is a biological application system for the clinical treatment of diseases and the research of life sciences, in which all kinds of human biological materials are stored intensively. ${ }^{1}$ The purpose of biobank is to collect biological information of specific population, and then to use these biological information to carry out medical scientific researches ${ }^{2}$. There is no doubt that biobanks play a very important role in medical scientific researches.

Biobanks have developed rapidly in recent years, and many countries have established their own biobanks. The large-scale and famous biobanks in the world mainly include UK Biobank, Danish National Biobank, Biobanks of the Biobanking and BioMolecular Resources Research In frastructure-European Research Infrastructure Consortium (BBMRI-ERIC), and China Kadoorie Biobank ${ }^{3-6}$. At the same time, there are more and more researches on biobank ${ }^{7,8}$. Biobanking research has become commonplace even in low- and middle-income countries (LMICs) ${ }^{9}$. China has established a national biobank since 1994. Since then, the 
construction of biobank has been booming in China. There are more and more biobanks in China, and some large-scale biobanks which is established by national clinical research centers appears. However, there are still many problems in the development of biobanks in China, such as small scale, few samples of healthy people, insufficient utilization, unshareable resources, backward information construction, and lack of relevant standards and operational specifications.

In this study, we will focus on a clinical biobank of a research-oriented hospital in China. In research-oriented hospital, clinical research is as important as clinical diagnosis and treatment, and clinical research is one of the most important tasks in the development of clinical disciplines. So, as an important platform and resource of clinical research, clinical biobank may play an important role in the development of clinical disciplines ${ }^{10}$. In the meantime, clinical disciplines may also play a key role in the development of clinical biobank. However, there is no research on the relationship of clinical biobank and clinical disciplines. Therefore, the purpose of this study is to understand the differences between different clinical disciplines in the development of clinical biobank, and to explore the relationship of clinical biobank and clinical disciplines.

\section{Methods}

\section{Study population}

This study was conducted in the Children's Hospital of Chongqing Medical University (CHCMU). CHCMU is one of two National Clinical Research Center for Child Health and Disorders in China. The survey subjects are mainly medical and scientific professionals in the hospital. And the survey is voluntary.

\section{Data collection}

A survey that focuses on knowledge, utilization and demand of biobank was conducted online. We released an online questionnaire in July 2019. The respondents filled out the items as required and submit them online. Finally, a total of 182 valid questionnaires were collected.

The questionnaire contains 32 questions in total, thirty of them are multiple choice questions, and the other two are fill-in-the-blank questions. The questions were grouped into four parts: (1) Demographic information includes department, professional titles, education, administrator and age. (2) Knowledge of the hospital's biobank which includes two parts. The first part is knowledge of biobank basic information, mainly including geographic location, management situation and WeChat public number of the biobank. The second part is knowledge of the special project of clinical sample resources, mainly including subsidy policy and sample warehousing process. Each part contains six questions, and each question has two answers: Yes and No. One score will be recorded when the answer is Yes, so the total score of each part is 6 scores, and the total score of all the two parts is 12 scores. (3) Status of utilization of the hospital's biobank, mainly including is there a person responsible for the collection and processing of samples in your biological sample project, do the samples currently collected by yourself have quality assurance, do you regularly monitor the quality of your samples. (4) Demand for the hospital's biobank, including willingness to pay to store samples in the hospital's biobank, is it acceptable to cost more than 5000 yuan per year for sample storage.

At the same time, we collected some indicators that can reflect the development of clinical disciplines, including the participation situation of National Natural Science Foundation of China (NSFC) and the number of published English papers in different clinical disciplines in the past five years (2014-2018). NSFC is the most common national fund project in China, so it is included in this study.

In this study, clinical disciplines were divided into key disciplines and non-key disciplines. And key disciplines are determined according to the evaluation results of discipline construction of government departments. 


\section{Data analysis}

SPSS 22.0 was used to complete data cleaning and analysis. Quantitative data was described by mean and standard deviation, and was analyzed by independent samples t test. But when the quantitative data did not conform to normal distribution, the rank sum test was used. Qualitative data was described by frequency and percentage, and was analyzed by chi-square test.

\section{Results}

\section{Basic information of sample}

A total of 182 valid questionnaires were collected. From the perspective of departments, most of the participants are from internal medicine, the proportion of internal medicine is $37.36 \%$. From the perspective of Professional titles, the proportion of junior, intermediate and senior professional titles is almost the same. From the perspective of education, about $80 \%$ of participants are doctors and masters. From the perspective of administrator, $23.08 \%$ of participants are administrator. From the perspective of age, more than half of the participants are under 40 years old. More details are shown in Table 1.

\section{Knowledge, utilization and demand of hospital's biobank}

In terms of knowledge of the hospital's biobank, $86.81 \%$ of people know that there is a hospital-level biobank in the hospital, but only $64.29 \%$ of people know the geographic location of the hospital's biobank, and only $40.66 \%$ of people know the WeChat public number of the hospital's biobank. $52.20 \%$ of people know about the special project of clinical sample resources in the hospital, but only $24.73 \%$ of people know the process of sample warehousing. In terms of utilization of the hospital's biobank, $59.89 \%$ of respondents said that there has people responsible for the collection and processing of samples in their biological sample project. $38.46 \%$ of people thought that samples currently collected by themselves had quality assurance. $42.31 \%$ of people would conduct regular quality monitoring on their own samples. In terms of demand for the hospital's biobank, $91.76 \%$ of the people are willing to pay to store their samples in the hospital's biobank. $33.52 \%$ of people think it is acceptable to cost more than 5000 yuan per year for sample storage. More details are shown in Table 2.

\section{comparison between different disciplines}

A total of 31 clinical disciplines participated in the survey. And there are 7 key disciplines in these 31 clinical disciplines, including four provincial key disciplines, and three university-level key disciplines. The survey participation rate of different clinical disciplines is shown in Table 3. As can be seen from Table 3, the participation rate of key disciplines is higher than that of non-key disciplines, but the difference is not statistically significant.

In terms of knowledge of the hospital's biobank, we calculated three scores as is shown in Table 4. We found that these three quantitative variables did not conform to normal distribution, so rank sum test was used to compare the differences between the two disciplines. The results show that key disciplines have higher score in knowledge of biobank basic information than non-key disciplines, and the difference is statistically significant. At the same time, key disciplines also have higher score in knowledge of the special project of clinical sample resources than non-key disciplines, although the difference is not statistically significant.

In terms of utilization of the hospital's biobank, we collected three related variables, and the results are shown in Table 5. As can be seen from Table 5, the proportion of people responsible for sample collection and processing in key disciplines is higher than that in non-key disciplines, and the difference is statistically significant. But there is almost no difference between key disciplines and non-key disciplines in terms of sample quality assurance and sample quality monitoring. 
Up to September 2019, the hospital's biobank has received biological samples of blood, tissue, sputum, cerebrospinal fluid and urine. According to statistics, the total number of samples is 63346, and 60077 of them are from key disciplines, Only 3269 of them are from non-key disciplines. The proportion of sample storage and sample delivery of key disciplines is $94.84 \%$ and $99.90 \%$ respectively, which are far higher than non-key disciplines. At the same time, key disciplines have higher sample delivery rate than non-key disciplines (Table 6).

In terms of demand for the hospital's biobank, although the difference is not statistically significant, the proportion of people who are willing to pay to store samples in key disciplines is higher than that in non-key disciplines. And the proportion of people who are willing to pay more than 5000 yuan per year for sample storage in key disciplines is also higher than that in non-key disciplines (Table 7).

In the aspect of discipline development, the participation situation of National Natural Science Foundation of China (NSFC) and the number of published English papers in different clinical disciplines in the past five years (2014-2018) are shown in Table 8. As can be seen from Table 8, although the number of NSFC applications, approvals and the number of published English papers in key disciplines is lower than that in non-key disciplines, the number of NSFC applications per capita, the approval rate of NSFC and the number of published English papers per capita in key disciplines are all higher than those in non-key disciplines.

\section{Discussion}

\section{Development of clinical biobank}

This study discusses the construction and development of biobank in research-oriented hospital from three aspects: doctors' knowledge, utilization and demand of biobank. In the aspect of knowledge of hospital's biobank, the overall awareness is good. But the awareness of sample warehousing process is low, less than a quarter of people know how to apply for sample warehousing. It shows that the hospital's biobank needs to strengthen propaganda work in the early stage of construction and development. In particular, more efforts should be made to publicize the procedures of sample warehousing and sample delivery. In the aspect of utilization of the hospital's biobank, there are still some problems need to be noticed. For example, only about a third of people thought the samples they collected so far were of good quality, and less than half of people would conduct regular quality monitoring on their own samples. Besides, the storage quantity of biological samples is relatively high, but the delivery quantity and delivery rate of biological samples is very low. Therefore, in the process of building biobank, the hospital's administrators should pay more attention to the quality of collected samples, and the standardized management of various samples is crucial for accessing high-quality biospecimens ${ }^{11}$. Besides, sample quality monitoring should be carried out regularly. After all, the quality of samples directly reflects the level of the development of biobank. At the same time, it is also necessary to improve sample delivery quantity and sample delivery rate, and it is very important to increase the use of samples. According to the survey results, this study also found that researchers are faced with many challenges such as insufficient staff for sample collection, insufficient funds, insufficient related equipment, insufficient professional training experience of staff, delayed delivery of collected samples in the process of biological sample collection. In the aspect of demand for the hospital's biobank, most people are willing to pay to store their samples in the hospital's biobank, but their willingness to pay is low. It is probably because most researchers lack sufficient funds for sample storage, so most researchers are not willingness to pay much cost to store their samples in the hospital's biobank.

Biobank is the solid basis of modern medical research, the foundation of the development of precision medicine, the source of translational medicine research ${ }^{12}$. The establishment of hospital level biobank is an important way to collect clinical samples of major diseases, which is of great significance ${ }^{13}$. However, the clinical biobank of the hospital still has deficiencies in workflow publicity, sample collection, sample transportation, sample storage, sample quality assurance and sample utilization. These problems are crucial and can not be ignored by the administrator of the biobank. Among these problems, sample utilization is 
particularly important, because sample utilization is one of the most important basic performance metrics of biobanks, and biobanks with low utilization rates can hardly achieve the goal of sustainable development ${ }^{14}$. So, these problems need to be continuously solved to improve the development level of the hospital's biobank, so that it can fully play its role in supporting clinical research.

\section{Development of clinical disciplines}

In terms of survey participation, the overall participation rate of key disciplines is higher than that of non-key disciplines, which indicates that key disciplines are more enthusiastic about the construction of the hospital's biobank.

In terms of knowledge of the hospital's biobank, the result suggests that the knowledge of the hospital's biobank in key disciplines is better than that in non-key disciplines. In terms of utilization of the hospital's biobank, key disciplines have more people responsible for sample collection and processing than non-key disciplines. In addition, key disciplines do better than non-key disciplines in sample storage quantity, sample delivery quantity and sample delivery rate. Therefore, it can be concluded that key disciplines make better use of the hospital's biobank than non-key disciplines. In terms of demand for the hospital's biobank, the willingness to store samples and the willingness to pay for sample storage of key disciplines are stronger than that of non-key disciplines. So, it is obvious that key disciplines have higher demand for the hospital's biobank than non-key disciplines. On the whole, the knowledge, utilization and demand on biobank of key disciplines are better than those of non-key disciplines. This may be related to the fact that key disciplines have abundant clinical biological sample resources and pay more attention to the construction of the hospital's biobank.

In the aspect of discipline development, the number of NSFC applications per capita, the approval rate of NSFC and the number of English articles per capita in key disciplines are all higher than those in nonkey disciplines. These results strongly illustrate that the scientific research and academic strength of key disciplines is stronger than that of non-key disciplines.

In summary, the construction and development of key disciplines is better than that of non-key disciplines. And key disciplines are the most important force in the construction and development of the hospital's biobank.

\section{The relationship of clinical biobank and clinical disciplines}

As an important platform for clinical research, biobank can provide a large number of rich and valuable biological sample resources for clinical research, thus providing important basic conditions for disease etiology exploration, precise prevention, early diagnosis and personalized treatment. Therefore, biobank is an important resource for discipline development. ${ }^{15}$ At the same time, discipline is also very important for the development of biobank. In particular, key disciplines play a critical role in the construction and development of biobank. If the clinical discipline of the hospital is well constructed, it will pay more attention to biological samples and have a higher demand for biological samples and biobank, which will be beneficial to promote the development of the hospital's biobank. When the clinical biobank has been well developed, it will become a very important resource for the development of clinical disciplines. So, this is a very good virtuous circle in the development of clinical biobank and clinical disciplines. In summary, the development of clinical biobank is closely related to the development of clinical disciplines.

Clinical biobank plays a critical role in the process of clinical discipline construction and development. Therefore, it can be a new breakthrough in the development of clinical disciplines. For key disciplines, they should continue to maintain their advantages in biological samples, make use of abundant biological sample resources, strengthen the research capacity of precision medicine and translational medicine, improve research efficiency, and continue to produce relevant research results, so as to promote the development of the disciplines to a higher level. For non-key disciplines, in order to achieve better development, discipline 
leaders should firstly call on medical and scientific professionals to pay more attention to the construction of clinical biobank. At the same time, it is critical to increase investment, and increase the collection and storage quantity of biological samples as much as possible. In addition, it is also important to strengthen the utilization of biological samples. Biological samples that have been stored in the biobank should be used in time to avoid making the biobank become a "waste biobank". The biobank will be an efficient biobank when most of its collected biospecimens are distributed to investigators, and the rapid distribution and use of biosamples can ensures that the intentions of the specimen donors have been honored ${ }^{16}$. If the non-key disciplines can improve the utilization rate of biological samples and give full play to the role of biological samples, it will be developed and improved, and the gap between key disciplines and non-key disciplines will be continuously narrowed. So non-key disciplines can catch up with and even surpass key disciplines by using clinical biobank.

In conclusion, clinical biobank is an important booster for the development of clinical disciplines. Clinical disciplines can take the clinical biobank as a new breakthrough for development, and make full use of the power of the clinical biobank to strive for the goal of building a world-class discipline.\{!!! INVALID CITATION !!! , \#0;Chen, 2011 \#63\}

\section{Acknowledgments}

We thank those colleagues who participated in this survey.

\section{Author Disclosure Statement}

No conflicting financial interests exist.

\section{References}

1. Simeon-Dubach D, Zeisberger S M, Hoerstrup S P. Quality Assurance in Biobanking for Pre-Clinical Research. Transfus Med Hemother 2016;43(5):353-357.

2. Yu K K, Zhang J, Li X, et al. Establishment and management of a lung cancer biobank in Eastern China. Thoracic Cancer 2015;58-63.

3. Fachiroh J, Dwianingsih E K, Wahdi A E, et al. Development of a Biobank from a Legacy Collection in Universitas Gadjah Mada, Indonesia: Proposed Approach for Centralized Biobank Development in Low-Resource Institutions. Biopreserv Biobank 2019.

4. Chen Z, Chen J, Collins R, et al. China Kadoorie Biobank of 0.5 million people: survey methods, baseline characteristics and long-term follow-up. Int J Epidemiol 2011;40(6):1652-66.

5. Trehearne A. Genetics, lifestyle and environment. UK Biobank is an open access resource following the lives of 500,000 participants to improve the health of future generations. Bundesgesundheitsblatt Gesundheitsforschung Gesundheitsschutz 2016;59(3):361-7.

6. Holub P, Swertz M, Reihs R, et al. BBMRI-ERIC Directory: 515 Biobanks with Over 60 Million Biological Samples. Biopreserv Biobank 2016;14(6):559-562.

7. Porteri C, Pasqualetti P, Togni E, et al. Public's attitudes on participation in a biobank for research: an Italian survey. BMC Medical Ethics 2014;15:81.

8. Bourgeois F T, Avillach P, Kong S W, et al. Development of the Precision Link Biobank at Boston Children's Hospital: Challenges and Opportunities. J Pers Med 2017;7(4).

9. Zawati M H, Tassé A M, Mendy M, et al. Barriers and Opportunities in Consent and Access Procedures in Low- and Middle-Income Country Biobanks: Meeting Notes from the BCNet Training and General Assembly. Biopreserv Biobank 2018;16(3):171-178. 
10. Quinlan P R, Mistry G, Bullbeck H, et al. A data standard for sourcing fit-for-purpose biological samples in an integrated virtual network of biobanks. Biopreserv Biobank 2014;12(3):184-91.

11. Zhou Y T, Ge X T, Shen Y F, et al. Follow-up of the manganese-exposed workers healthy cohort (MEWHC) and biobank management from 2011 to 2017 in China. BMC Public Health 2018;18:944.

12. Draanen G V, Davidson P, Bour-Jordan H, et al. Assessing Researcher Needs for a Virtual Biobank. Biopreserv Biobank 2017;15(3):203-210.

13. Lacerda E M, Mudie K, Kingdon C C, et al. The UK ME/CFS Biobank: A Disease-Specific Biobank for Advancing Clinical Research Into Myalgic Encephalomyelitis/Chronic Fatigue Syndrome. Front Neurol 2018;9:1026.

14. Henderson M K, Goldring K, Simeon-Dubach D, et al. Advancing Professionalization of Biobank Business Operations: Performance and Utilization. Biopreserv Biobank 2019;17(3):213-218.

15. Hirata M, Kamatani Y, Nagai A, et al. Cross-sectional analysis of BioBank Japan clinical data: A large cohort of 200,000 patients with 47 common diseases. J Epidemiol 2017;27(3S):S9-S21.

16. Grizzle W E, Sexton K C, McGarvey D, et al. Lessons Learned During Three Decades of Operations of Two Prospective Bioresources. Biopreserv Biobank 2018;16(6):483-492.

\section{Tables}

\section{Table 1. Sample composition}

\begin{tabular}{lcc}
\hline Variable & Variable & Fre \\
\hline Do you know that there is a hospital-level biobank in our hospital? & Yes & 15 \\
& No & 24 \\
Do you know the geographic location of the hospital's biobank? & Yes & 11 \\
& No & 65 \\
Do you know the WeChat public number of the hospital's biobank? & Yes & 74 \\
& No & 10 \\
Do you know the special project of clinical sample resources? & Yes & 95 \\
& No & 87 \\
Do you know how to apply for sample warehousing? & Yes \\
& No \\
Is there a person responsible for the collection and processing of samples in your biological sample project? & Yes \\
& No & 10 \\
Do the samples currently collected by yourself have quality assurance? & Yes \\
& No & 70 \\
Do you regularly monitor the quality of your samples? & Yes \\
& No & 77 \\
Are you willing to pay to store samples in the hospital's biobank? & Yes & 16 \\
& No & 15 \\
Can you accept sample storage costs of more than 5000 yuan per year? & Yes \\
Total & No & 12 \\
& Total & 18 \\
\hline
\end{tabular}

Table 2. Knowledge, utilization and demand of hospital's biobank

Table 3. Survey participation rate of different clinical disciplines

\begin{tabular}{lllll}
\hline Clinical Disciplines & Number of Participants & Participation Rate(\%) & $P$ \\
\hline Key Disciplines & 44 & 22.45 & 3.684 & 0.055
\end{tabular}




\begin{tabular}{llll}
\hline Clinical Disciplines & Number of Participants & Participation Rate(\%) & $P$ \\
\hline Non-key Disciplines & 138 & 16.63 & \\
Total & 182 & 17.74 & \\
\hline
\end{tabular}

Table 4. Knowledge of the hospital's biobank

\begin{tabular}{llllll}
\hline Variables & $\begin{array}{l}\text { Key } \\
\text { Disciplines }\end{array}$ & $\begin{array}{l}\text { Non-key } \\
\text { Disciplines }\end{array}$ & Total & & $P$ \\
\hline $\begin{array}{l}\text { Willing to pay to } \\
\text { store samples }\end{array}$ & $42(95.45$ & $125(90.58$ & $167(91.76$ & 0.503 & 0.478 \\
$\begin{array}{l}\text { Willing to pay } \\
\text { more than }\end{array}$ & $18(40.91$ & $43(31.16$ & $61(33.52$ & 1.423 & 0.233 \\
$\begin{array}{l}5000 \text { yuan per } \\
\text { year for } \\
\text { sample storage }\end{array}$ & & & & \\
\hline
\end{tabular}

Table 5. Status of utilization of the hospital's biobank

Table 6. Sample storage status

\begin{tabular}{llll}
\hline Clinical Disciplines & $\begin{array}{l}\text { Number of sample } \\
\text { storage }\end{array}$ & Sample delivery quantity & Sample delivery rate(\%) \\
\hline Key Disciplines & 60077 & 5042 & 8.39 \\
Non-key Disciplines & 3269 & 5 & 0.15 \\
Total & 63346 & 5047 & 7.97 \\
\hline
\end{tabular}

Table 7. Demand for the hospital's biobank

Table 8. The participation situation of NSFC and the number of published English papers in different clinical disciplines in the past five years (2014-2018)

\begin{tabular}{llll}
\hline Clinical Disciplines & the number of NSFC applications & the number of NSFC applications per capita & the number of NS \\
\hline Key Disciplines & 153 & 0.78 & 28 \\
Non-key Disciplines & 348 & 0.42 & 55 \\
Total & 501 & 0.49 & 83 \\
\hline
\end{tabular}

